\title{
Desenvolvimento de Nasonia vitripennis (Walker, 1836) (Hymenoptera: Pteromalidae) em pupas de Cochliomyia macellaria (Fabricius, 1775) (Diptera: Calliphoridae), utilizando diferentes densidades do parasitóide
}

\author{
Leandro Silva Barbosa ${ }^{1,3}$; Márcia Souto Couri ${ }^{1}$, Valéria Magalhães Aguiar Coelho \\ ${ }^{1}$ Laboratório de Diptera, Departamento de Entomologia, Museu Nacional, \\ Quinta da Boa Vista, CEP 20940-040 Rio de Janeiro, RJ, Brasil \\ ${ }^{2}$ Laboratório de Estudo de Dípteros, Departamento de Microbiologia e Parasitologia, \\ Universidade Federal do Estado do Rio de Janeiro - UNIRIO, \\ Rua Frei Caneca, 94, CEP 20211-040, Rio de Janeiro, RJ, Brasil \\ ${ }^{3}$ Autor para correspondência: Leandro Silva Barbosa, e-mail: leanbarbosa@globo.com
}

Barbosa, L. S.; Couri, M. S.; Aguiar-Coelho, V. M. Development of Nasonia vitripennis (Walker, 1836) (Hymenoptera: Pteromalidae) in pupae of Cochliomyia macellaria (Fabricius, 1775) (Diptera: Calliphoridae), using different densities of parasitoid. Biota Neotrop., vol. 8, no. 1, Jan./Mar. 2008. Available from: <http:// www.biotaneotropica.org.br/v8n1/en/abstract?article+bn00808012008>.

\begin{abstract}
The post-embryonic development, the productivity of the pupae, the rate of parasitism and the sexual ratio of Nasonia vitripennis (Walker) reared in pupae of Cochliomyia macellaria (Fabricius) were studied. Different densities of the parasitoid were used (proportions 1:1, 2:1, 3:1, 4:1, and 5:1) with exposition of 72 hours. Nulliparous females originating from the stock colony were individualized in test tubes covered with hydrophobic cotton and containing the host pupae. Each treatment was constituted by 10 repetitions. After the exposition, the wasps were discarded and the host pupae were individualized in test tubes until the emergency of the adults of $C$. macellaria or N. vitripennis. Samples of host pupae not exposed to parasitism and receiving the same experimental treatment were used as control. The post-embryonic period was significantly shorter in the proportion of 2:1 (13,73 days) and longer in the relation of 5:1. The peaks of emergence occurred in the $14^{\text {th }}$ day after the exposition of the parasitoid to the host in all relations, except for the relation of $4: 1\left(13^{\text {rd }}\right.$ day). The productivity of $N$. vitripennis does not vary significantly among the different proportions. As the number of parasitoids grows, its post-embryonic development gets slower and the sexual ratio of males grows up. The rate of parasitism showed a decrease when the host was exposed to five parasitoids.
\end{abstract}

Keywords: biological control, wasp, fly, parasitism.

Barbosa, L. S.; Couri, M. S.; Aguiar-Coelho, V. M. Desenvolvimento de Nasonia vitripennis (Walker, 1836) (Hymenoptera: Pteromalidae) em pupas de Cochliomyia macellaria (Fabricius, 1775) (Diptera: Calliphoridae), utilizando diferentes densidades do parasitóide. Biota Neotrop., vol. 8, no. 1, jan./mar. 2008. Disponível em: <http://www.biotaneotropica.org.br/v8n1/pt/abstract?article+bn00808012008>.

Resumo: O desenvolvimento pós-embrionário, a produtividade da pupa, a taxa de parasitismo e a razão sexual de Nasonia vitripennis (Walker) criadas em pupas de Cochliomyia macellaria (Fabricius) foram estudadas. Diferentes densidades do parasitóide foram utilizadas (proporções 1:1, 2:1, 3:1, 4:1 e 5:1) com exposição de 72 horas. Fêmeas nulíparas originárias da colônia estoque foram individualizadas em tubos de ensaio cobertos com algodão hidrofóbico, contendo uma pupa hospedeira. Cada tratamento foi constituído de 10 repetições. Após a exposição, as vespas foram descartadas e as pupas hospedeiras foram individualizadas em tubos de ensaio até a emergência dos adultos de C. macellaria ou $N$. vitripennis. Amostras de pupas hospedeiras não expostas ao parasitismo e recebendo o mesmo tratamento experimental foram utilizadas como controle. O período pósembrionário foi significativamente mais curto na proporção 2:1 (13,73 dias) e mais longo na relação 5:1. Os picos de emergência ocorreram no $14^{\circ}$ dia após a exposição do parasitóide ao hospedeiro para todas as relações, com exceção da relação 4:1(13 ${ }^{\circ}$ dia). A produtividade de $N$. vitripennis não variou significativamente entre as diferentes proporções. Conforme o número de parasitóides aumentou foi observado uma maior duração do período do desenvolvimento pós-embrionário e um aumento na proporção de machos na prole. A taxa de parasitismo apresentou queda quando o hospedeiro foi exposto a cinco parasitóides.

Palavras-chave: controle biológico, vespa, mosca, parasitismo. 


\section{Introdução}

Programas de controle biológico de pragas têm apresentado grande crescimento devido ao novo direcionamento internacional de produção agropecuária, favorecendo a conservação e o uso sustentável dos recursos biológicos, requisitos básicos da Convenção da Biodiversidade. A utilização de inimigos naturais no controle de pragas tem se mostrado uma alternativa promissora devido à eficiência e aos baixos custos relativos.

Nasonia vitripennis (Walker, 1836) (Hymenoptera: Pteromalidae) é uma espécie de micro-vespa parasitóide gregária, pois em um único hospedeiro pode ocorrer o desenvolvimento de vários indivíduos. São polífagas, podendo parasitar uma enorme gama de espécies, Whiting (1967) apontou em sua revisão 68 espécies de hospedeiros. E atualmente vêm sendo descobertas novas espécies hospedeiras (Marchiori et al. 2001). Também podem produzir larvas diapáusicas (Whiting 1967) dependendo de fatores ambientais tais como: temperatura, fotoperíodo, umidade, espécie hospedeira e idade da fêmea parasitóide (Wylie 1963, Walker \& Pimentel 1966, Saunders et al. 1970), podendo produzir assim, uma ação mais prolongada no local onde for aplicada. Devido a essas características, esta espécie possui vantagens para ser usada em programas de controle biológico aplicado de dípteros muscóides.

Esse parasitóide é cosmopolita (Rueda \& Axtell 1985) e a sua taxa de reprodução pode ser potencializada com a utilização de pupários de maior porte. Tais observações justificam a utilização de representantes das famílias Calliphoridae e Sarcophagidae como hospedeiros preferenciais, porém, podem também atacar integrantes da família Muscidae, cujo pupário geralmente é menor (Harvey \& Gols 1998, Cardoso \& Milward-de-Azevedo 1995).

Cochliomyia macellaria (Fabricius, 1775) (Diptera: Calliphoridae) é vulgarmente conhecida como mosca-varejeira, é a principal espécie causadora de miíase secundária no Brasil. Devido ao hábito necrófago das suas larvas, este díptero geralmente se encontra associado com outras espécies de califorídeos, sarcofagídeos e muscídeos. Aliado a isto, é veiculadora mecânica de diversos agentes patogênicos, apresentando importância médica, veterinária e econômica (Baumgartner \& Greenberg 1985). No Brasil, assume relevância maior em virtude de ser uma das espécies veiculadoras de ovos de Dermatobia hominis (Linnaeus Jr, 1781) (Diptera: Oestridae), mosca responsável pela miíase furuncular ou dermatobiose que causa muitos prejuízos à pecuária nacional (Guimarães et al. 1983, Moya-Borja 2003).

O presente trabalho tem por objetivo avaliar a duração do período pós-embrionário, produtividade de parasitóides por hospedeiro, razão sexual e taxa de parasitismo de $N$. vitripennis criadas em pupas de C. macellaria em diferentes densidades do parasitóide.

\section{Material e Métodos}

O estabelecimento da colônia de C. macellaria foi realizado através de coleta em área rural, na cidade de Paracambi no Rio de Janeiro. A coleta foi realizada com auxílio de armadilha típica para coleta de lepidópteros, na qual foi adaptada uma manga para facilitar a captura dos dípteros vivos (Barbosa 2006). Como isca utilizou-se sardinha com 24 horas de exposição ao ambiente. Para a identificação dos exemplares utilizou-se a chave taxonômica de Mello (2003).

A colônia estoque de $C$. macellaria foi mantida de acordo com a metodologia preconizada por Cunha-e-Silva \& Milward-de-Azevedo (1994) e Paes \& Milward-De-Azevedo (1998). Visando a manutenção da colônia estoque de $N$. vitripennis, pupas de $C$. macellaria, oriundas da colônia estoque, com no máximo 24 horas, foram acondicionadas em sacos plásticos em lotes de aproximadamente 150 unidades e congeladas em refrigerador. Cada lote foi identificado com a data de congelamento.
A colônia estoque de $N$. vitripennis foi estabelecida a partir de coletas realizadas no Jardim Zoológico do Rio de Janeiro (Fundação Rio-Zoo). Para captura dos parasitóides foi utilizada uma gaiola própria para criação de moscas com aproximadamente $45 \mathrm{~cm}^{3}$, cuja tela permitia a entrada dos parasitóides, porém evitava a predação das pupas por outros animais. No interior das mesmas, como iscas, foram expostas pupas de Chrysomya megacephala (Fabricius, 1794) com aproximadamente 24 horas de idade provenientes de colônia estoque e carne bovina putrefata como fonte de apneumônio. Dois dias após a exposição no campo, as pupas foram recolhidas e acondicionadas em frascos de vidro transparentes ( $13 \mathrm{~cm}$ de diâmetro $\times 24 \mathrm{~cm}$ de altura) vedados com tecido de algodão até a emergência dos parasitóides.

A identificação dos parasitóides recém-emergidos foi realizada por meio da descrição taxonômica detalhada de Rueda \& Axtell (1985) e confirmada pela especialista no grupo Dra. Maria Angélica Penteado Dias (Departamento de Ecologia e Biologia Evolutiva da Universidade de São Carlos, São Paulo). A colônia estoque dos microhimenópteros foi mantida em câmara climatizada regulada a $25^{\circ} \mathrm{C}$, $60 \pm 10 \%$ de umidade relativa do ar e 14 horas de fotofase, de acordo com metodologia preconizada por Cardoso \& Milward-de-Azevedo (1996). Os adultos foram mantidos em gaiolas confeccionadas com recipiente plástico de um litro. A alimentação foi realizada com oferta de solução de mel e água (1:1), embebida em algodão dentro de um recipiente plástico com $4 \mathrm{~cm}$ de diâmetro.

Pupas de C. macellaria previamente congeladas por no máximo dois meses, foram depositas em papel filtro e descongeladas, com auxílio de uma luminária incidindo sobre as mesmas por $10 \mathrm{mi}-$ nutos. As pupas, distribuídas em placas de Petri teladas com filó, foram expostas aos parasitóides recém emergidos, por períodos de 48 horas. Após a exposição, foram alocadas em recipientes cilíndricos cobertos com tecido de náilon (escaline), identificado com geração, data, período de exposição e provável data de emergência, e mantidos em câmara climatizada, onde se aguardava a emergência da nova geração.

A etapa experimental foi conduzida em câmara climatizada (Quimis) regulada a $27{ }^{\circ} \mathrm{C}, 60 \pm 10 \%$ de umidade relativa do ar e 14 horas de fotofase. A fotofase foi iniciada às 6:00 horas da manhã. Foram utilizadas fêmeas nulíparas de $N$. vitripennis pertencentes a $23^{\mathrm{a}}$ geração, com até 24 horas de idade, medindo de 2 a $3 \mathrm{~mm}$ e previamente alimentadas com solução de água e mel (2:1). Como hospedeiros, utilizaram-se pupas frescas de $C$. macellaria oriundas da $19^{a}$ geração, com até 24 horas de idade e com peso corporal registrado entre 48 e $49 \mathrm{mg}$. Lotes de uma, duas, três, quatro ou cinco fêmeas parasitóides nulíparas provenientes da colônia estoque foram individualizadas em tubos de ensaio com $50 \mathrm{~mL}$ de capacidade e tampados com algodão hidrófobo, contendo uma pupa hospedeira. Cada tratamento foi constituído por 10 repetições. No interior de cada tubo inseriu-se uma tira de papel de filtro embebido em solução de água e mel (2:1). Após 72 horas de exposição, as vespas foram descartadas e as pupas hospedeiras individualizadas em tubos de ensaio, distribuídos aleatoriamente em bandejas onde se aguardou a emergência dos adultos de C. macellaria ou N. vitripennis.

Lotes de pupas hospedeiras não expostas ao parasitismo, apresentando o mesmo delineamento experimental, foram utilizadas como controle para verificar a taxa de mortalidade natural. As observações foram feitas diariamente, sob microscópio estereoscópico (LEICA) sempre no mesmo horário até o $35^{\circ}$ dia após a exposição. Como parâmetros de comparação foram usados dados referentes à duração do desenvolvimento pós-embrionário, produtividade de parasitóides por hospedeiro, razão sexual e taxa de parasitismo. Considera-se a produtividade por hospedeiro o número de parasitóides emergidos por pupa hospedeira, enquanto a taxa de parasitismo o número de pupas de C. macellaria que originaram parasitóides. 
Para a análise bruta dos dados e elaboração dos gráficos utilizouse o programa Microsoft ${ }^{\circledR}$ Excel 2002. Para avaliar o significado estatístico dos resultados foram utilizados a Análise de Variância e o Teste de Tukey através do programa GraphPad Prism 4.03 (Magalhães \& Lima 2001).

\section{Resultados}

A duração média do desenvolvimento pós-embionário de machos e fêmeas de $N$. vitripennis criadas em diferentes densidades variou significativamente pela análise de variância $(\mathrm{P}<0,0001$ e $\mathrm{F}=12,47)$. $\mathrm{O}$ período pós-embrionário mais curto foi observado na densidade 2:1 (13,73 dias), diferindo significativamente pelo teste $\mathrm{T}$ das demais relações (Tabela 1). O mais longo foi na relação 5:1, diferindo significativamente das relações menos elevadas, com exceção da 1:1. Com relação ao sexo masculino observou-se um aumento significativo na duração do período pós-embrionário na relação 5:1 comparando-se com as demais densidades. As fêmeas não apresentaram a mesma tendência (Tabela 1).

O ritmo de emergência de $N$. vitripennis teve pico no $14^{\circ}$ dia após a exposição do parasitóide ao hospedeiro para todas as relações, com exceção da relação 4:1 (Figura 1), cujo pico foi no $13^{\circ}$ dia. A produtividade de $N$. vitripennis criadas em pupas de $C$. macellaria utilizando-se diferentes densidades de parasitóide: hospedeiro (1:1, $2: 1,3: 1,4: 1$ e $5: 1)$ não variou significativamente pela ANOVA $(\mathrm{F}=0,47$ e $\mathrm{P}=0,75)$ (Tabela 2). O gráfico de tendência exponencial mostrou uma queda na emergência de fêmeas com o aumento das densidades (Figura 2).

Observou-se uma taxa de parasitismo de $N$. vitripennis em C. macellaria de $100 \%$ em todas as relações só decrescendo na densidade 5:1, onde em 10\% das pupas não emergiram parasitóides nem dípteros (Figura 3). Os controles relativos às densidades de $1: 1,2: 1$ e $3: 1$ apresentaram a taxa de emergência de dípteros de $100 \%$. Já as relações de 4:1 e 5:1 apresentaram taxa de emergência de dípteros de $98 \%$.

\section{Discussão}

Os resultados apresentados mostraram uma tendência à extensão da duração do desenvolvimento pós-embrionário em função do aumento do número de parasitóides por hospedeiro. Cardoso \& Milward-de-Azevedo (1995), analisaram a influência do aumento da densidade de $C$. megacephala sobre o desenvolvimento de $N$. vitripennis, observaram que o aumento do número de hospedeiros, também propiciou a extensão da duração do desenvolvimento pósembrionário, apesar de não referida pelos autores. (Barbosa 2006) verificou o mesmo efeito estudando a exposição de crescentes números de hospedeiros C. macellaria sob o parasitóide $N$. vitripennis. Esses resultados, apesar de semelhantes, procedem de mensurações antagonistas, logo se sugere que o desenvolvimento desse parasitóide é influenciado pela sua densidade, onde existe uma amplitude ótima. Densidades além desta faixa, tanto para cima como para baixo, podem alongar o período pós-embrionário.

Segundo Slansky \& Scriber (1985), o melhor desempenho de insetos gregários é obtido em uma faixa de densidade particular, ocorrendo declínio acima ou abaixo deste intervalo, devido a indução de condições micro-ambientais não favoráveis ao desenvolvimento destes insetos. O desenvolvimento mais lento de $N$. vitripennis na densidade 5 parasitóides: 1 hospedeiro em relação as menores densidades (1:1, 2:1, 3:1 e 4:1), provavelmente ocorreu devido ao superparasitismo, em que o número de parasitóides presentes em um único hospedeiro provavelmente foi tão elevado, favorecendo a competição, que nem o parasitóide e nem o hospedeiro emergiram, induzindo a uma taxa de inviabilidade de pupas (Figura 3). Devido à ausência
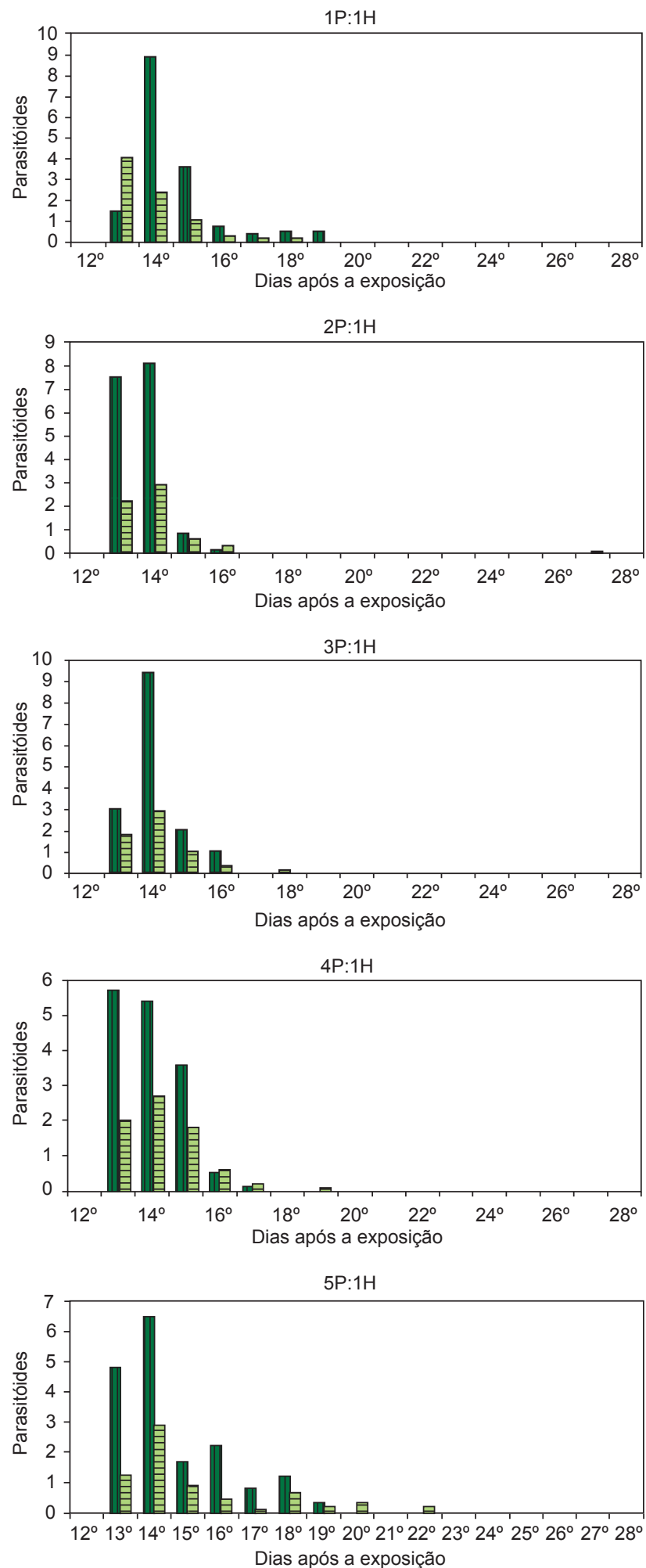

Figura 1. Ritmo de emergência de fêmeas e machos de Nasonia vitripennis criadas em pupas de Cochliomyia macellaria expostas por 72 horas a fêmeas nulíparas utilizando-se diferentes relações de fêmeas parasitóides/hospedeiro $\left(27^{\circ} \mathrm{C}, 60 \pm 10 \%\right.$ U.R. e 14 horas de fotofase $\mathrm{P}=$ parasitóide, $\mathrm{H}=$ hospedeiro), em laboratório.

Figure 1. Emergence rhythm of females and males of Nasonia vitripennis reared in pupae of Cochliomyia macellaria exposed per 72 hours for nulliparous females, using different relations of parasitoid females/host $\left(27^{\circ} \mathrm{C}\right.$, $60 \pm 10 \%$ U.R. and 14 hours of fotofase $\mathrm{P}=$ parasitoid, $\mathrm{H}=$ host), in laboratory. 
Barbosa, LS. et al.

Tabela 1. Duração média do desenvolvimento ontogenético (em dias) de Nasonia vitripennis criadas em pupas de Cochliomyia macellaria expostas ao parasitismo por 72 horas, utilizando-se diferentes relações parasitóide $1 /$ hospedeiro $2\left(27{ }^{\circ} \mathrm{C}, 60 \pm 10 \%\right.$ U.R. e 14 horas de fotofase), em laboratório.

Table 1. Average of ontogenetic development (days) of Nasonia vitripennis reared in pupae of Cochliomyia macellaria exposed per 72 hours to parasitism, using different relations of parasitoid females/host $\left(27^{\circ} \mathrm{C}, 60 \pm 10 \%\right.$ U.R. and 14 hours of fotofase), in laboratory.

\begin{tabular}{|c|c|c|c|c|c|c|c|c|c|}
\hline \multirow{3}{*}{$\begin{array}{c}\text { Relação } \\
\text { Parasitóide: } \\
\text { Hospedeiro }\end{array}$} & \multicolumn{9}{|c|}{ Duração do período pós-embrionário (dias) } \\
\hline & \multicolumn{3}{|c|}{ Machos } & \multicolumn{3}{|c|}{ Fêmeas } & \multicolumn{3}{|c|}{ Machos e Fêmeas } \\
\hline & $\mathbf{X}$ & $\pm \mathbf{d} s$ & IV & $\mathbf{X}$ & $\pm s$ & IV & $\mathbf{X}$ & $\pm s$ & IV \\
\hline $1: 1$ & $13,77_{b}$ & 0,12 & $13-18$ & $14,54_{a}$ & 1,25 & 13-19 & 14,28 & 1,23 & $13-19$ \\
\hline $2: 1$ & $14,05_{\mathrm{b}}$ & 1,87 & $13-27$ & $13,61_{b}$ & 0,61 & $13-16$ & $13,73_{\mathrm{b}}^{\mathrm{c}}$ & 1,11 & $13-27$ \\
\hline $3: 1$ & $13,97_{b}^{\circ}$ & 0,82 & $13-16$ & $14,09^{\circ}$ & 0,82 & $13-18$ & $14,06^{\circ}$ & 0,82 & $13-18$ \\
\hline $4: 1$ & $14,28_{\mathrm{b}}$ & 1,16 & $13-19$ & $13,95_{c}$ & 0,89 & $13-17$ & $14,06_{\mathrm{a}}$ & 1,00 & $13-19$ \\
\hline $5: 1$ & $15,21_{\mathrm{a}}$ & 2,32 & $13-22$ & $14,60_{\mathrm{a}}$ & 1,60 & $13-19$ & $14,49_{\mathrm{c}}$ & 1,66 & $13-22$ \\
\hline
\end{tabular}

$\mathrm{X}=$ média, $\mathrm{d} \mathrm{s}=$ desvio padrão, $\mathrm{IV}=$ intervalo de variação; Médias seguidas pela mesma letra não diferem significativamente pelo teste $\mathrm{T}$ em nível de $5 \%$ de significância. ${ }^{1}$ Fêmeas nulíparas individualizadas $\left(n=10\right.$ /tratamento) com até 24 horas pós-emergência; ${ }^{2}$ Pupas com até 24 horas de idade.

$\mathrm{X}=$ average, ds= standart desviation, IV= Interval Scale; Averages followed by the same letter don't differ by the T test in the 5\% of significance level.

${ }^{1}$ Nulliparous individualized females $\left(n=10 /\right.$ treatment) with at most 24 hours of emergence. ${ }^{2}$ Pupae with at last 24 hours age.

Tabela 2. Razão sexual e número de parasitóides adultos de Nasonia vitripennis criados em pupas de Cochliomyia macellaria expostas ao parasitismo por 72 horas, utilizando-se diferentes relações parasitóide ${ }^{1 / h}$ hospedeiro ${ }^{2}\left(27^{\circ} \mathrm{C}, 60 \pm 10 \%\right.$ U.R. e 14 horas de fotofase), em laboratório.

Table 2. Sex ratio and number of adults of Nasonia vitripennis reared in pupae of Cochliomyia macellaria exposed per 72 hours, using different relations of parasitoid females/host $\left(27^{\circ} \mathrm{C}, 60 \pm 10 \%\right.$ U.R. and 14 hours of fotofase $)$, in laboratory.

\begin{tabular}{|c|c|c|c|c|c|c|c|}
\hline \multirow{3}{*}{$\begin{array}{c}\text { Relação } \\
\text { Parasitóide: } \\
\text { Hospedeiro }\end{array}$} & \multirow[t]{3}{*}{ Razão sexual } & \multicolumn{6}{|c|}{ Número de parasitóides por hospedeiro } \\
\hline & & \multicolumn{2}{|c|}{ Machos } & \multicolumn{2}{|c|}{ Fêmeas } & \multicolumn{2}{|c|}{ Total } \\
\hline & & $\mathbf{X}$ & IV & $\mathbf{X}$ & IV & $\mathbf{X}$ & IV \\
\hline $1: 1$ & 0,75 & $7,77_{\mathrm{a}}$ & $2-30$ & $17,75_{\mathrm{a}}$ & $13-24$ & $23,56_{a}$ & $16-30$ \\
\hline $2: 1$ & 0,81 & $6,10_{\mathrm{a}}$ & $1-10$ & $18,33_{\mathrm{a}}$ & $10-23$ & $22,60_{a}$ & $1-31$ \\
\hline $3: 1$ & 0,72 & $6,00_{\mathrm{a}}$ & $2-10$ & $15,50_{a}$ & $9-21$ & $21,50_{\mathrm{a}}$ & $12-28$ \\
\hline $4: 1$ & 0,67 & $8,22_{\mathrm{a}}$ & $3-17$ & $15,30_{\mathrm{a}}$ & $1-24$ & $22,70_{a}$ & $1-33$ \\
\hline $5: 1$ & 0,71 & $7,87_{\mathrm{a}}$ & $2-12$ & $17,44_{a}$ & $8-27$ & $24,44_{a}$ & $8-35$ \\
\hline
\end{tabular}

Razão sexual = média de fêmeas /média do total, $\mathrm{X}=$ média, IV = intervalo de variação; Médias seguidas pela mesma letra não diferem significativamente pelo teste T em nível de 5\% de significância. ${ }^{1}$ Fêmeas nulíparas individualizadas ( $\mathrm{n}=10 /$ tratamento) com até 24 horas pós-emergência; ${ }^{2}$ Pupas com até 24 horas de idade.

Sexual ratio $=$ female average/total average, $X=$ average, $I V=$ Interval Scale. Averages followed by the same letter don't differ by the T test in the $5 \%$ of significance level. ${ }^{1}$ Nulliparous individualized females $\left(n=10 /\right.$ treatment) with at most 24 hours of emergence. ${ }^{2}$ Pupae with at last 24 hours age.

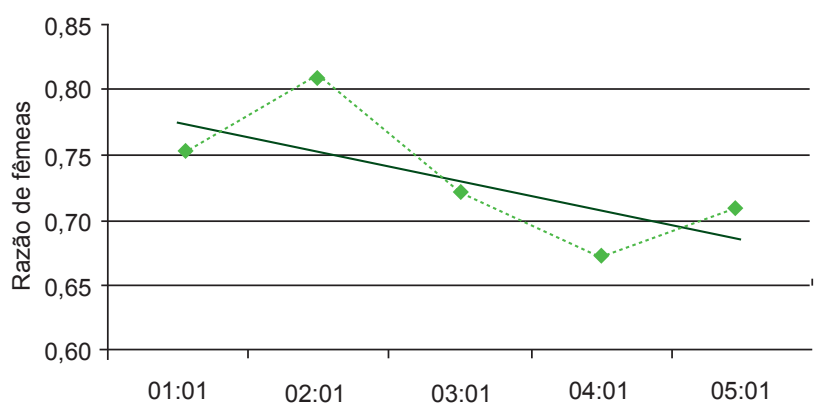

Relação parasitóide: hospedeiro

_-... Porcentagem de fêmeas na prole
Expon. (Porcentagem de fêmeas na prole)

Figura 2. Gráfico de tendência exponencial da taxa de fêmeas de Nasonia vitripennis criadas em pupas de Cochliomyia macellaria expostas por 72 horas a fêmeas nulíparas, utilizando-se diferentes relações de fêmeas parasitóides/hospedeiro $\left(27^{\circ} \mathrm{C}, 60 \pm 10 \%\right.$ U.R. e 14 horas de fotofase), em laboratório.

Figure 2. Exponencial tendency graphic of female ratio of Nasonia vitripennis reared in pupae of Cochliomyia macellaria exposed per 72 hours for nulliparous females, using different relations of parasitoid females/ host $\left(27^{\circ} \mathrm{C}\right.$, $60 \pm 10 \%$ U.R. and 14 hours of fotofase), in laboratory.

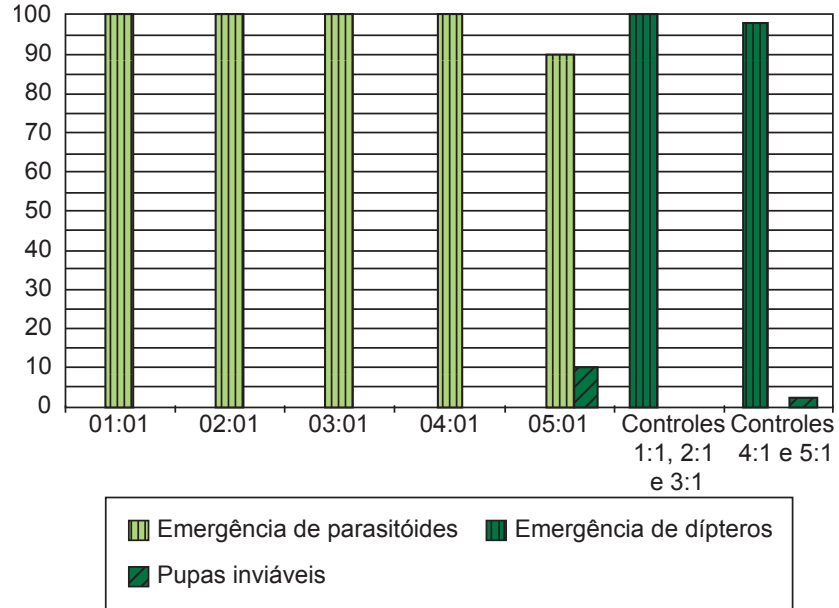

Figura 3. Porcentagem de parasitoidismo de Nasonia vitripennis criadas em pupas de Cochliomyia macellaria expostas por 72 horas a fêmeas nulíparas, utilizando-se diferentes relações de fêmeas parasitóides ${ }^{1 / h o s-}$ pedeiro ${ }^{2}\left(27^{\circ} \mathrm{C}, 60 \pm 10 \%\right.$ U.R. e 14 horas de fotofase), em laboratório. ${ }^{1}$ Fêmeas nulíparas individualizadas $(\mathrm{n}=10 /$ tratamento) com até 24 horas pós-emergência. ${ }^{2}$ Pupas com até 24 horas pós-emergência.

Figure 3. Parasitoidism ratio of Nasonia vitripennis reared in pupae of Cochliomyia macellaria exposed per 72 hours for nulliparous females, using different relations of parasitoid females ${ }^{1} /$ host $^{2}\left(27^{\circ} \mathrm{C}, 60 \pm 10 \%\right.$ U.R. and 14 hours of fotofase), in laboratory. ${ }^{1}$ Individualized nulliparous females ( $n=10 /$ treatment) with at most 24 hours after hatch. ${ }^{2}$ Pupae with at most 24 hours after hatch. 
de terminologia para categorizar o mesmo efeito de retardamento decorrente de baixas densidades, propõe-se o termo subparasitismo que abrangerá os efeitos deletérios causados ao desenvolvimento de parasitóides gregários.

Os picos de emergência para machos e para fêmeas, no presente trabalho, obedeceram à mesma tendência entre si, diferindo apenas na relação 1:1, na qual foi observado um pico de emergência de machos precedendo ao de fêmeas. Durações de desenvolvimento similares entre machos e fêmeas foram constatados por Cardoso \& Milward-de-Azevedo (1995).

A produtividade média dos parasitódes por pupa variou entre 21,50 (3:1) a 24,44 (5:1). Não foi observada diferença significativa na produtividade de parasitóides com o aumento do número de $N$. vitripennis expostas a uma pupa hospedeira, possivelmente em virtude das amplitudes trabalhadas. Porém, os machos foram mais influenciados do que as fêmeas, pelas diferentes densidades, pois apresentaram um aumento significativo da duração do desenvolvimento pós-embrionário com o aumento do número de parasitóide. Sugere-se que os machos são mais sensíveis às mudanças ambientais do microhabitat hospedeiro. Acredita-se que essa diferença entre os sexos se deva principalmente ao menor tamanho do macho, que influi tanto no calor metabólico como nas suas necessidades nutricionais. O desenvolvimento do parasitóide está intimamente associado ao tamanho e qualidade do hospedeiro (Cardoso \& Milward-de-Azevedo 1996, Harvey \& Gols 1998), bem como na densidade de parasitóides e tempo de exposição do hospedeiro, já que esse influi no tamanho da prole deixada (Cardoso \& Milward-de-Azevedo 1995).

O gráfico de tendência exponencial mostrou que a taxa de fêmeas na prole caiu devido ao aumento do número de parasitóides por hospedeiro. Essa mesma tendência foi observada por Cardoso \& Milward-de-Azevedo (1995) em função do aumento de hospedeiros. Muitas teorias vêm sendo apresentadas sobre a razão sexual em parasitóides. Wylie (1966) sugere que o superparasitismo é um dos fatores diretamente responsáveis pelo aumento da porcentagem de machos na prole, corroborando o presente estudo.

Harvey \& Gols (1998) observaram uma variação da razão sexual de Muscidifurax raptorellus Kogan \& Legner (Hymenoptera: Pteromalidae) em função do tamanho e da espécie do hospedeiro. Estes autores verificaram que proles oriundas de pupas pequenas de Musca domestica Linnaeus (Diptera: Muscidae) originaram um número maior de fêmeas, enquanto as pupas pequenas de Calliphora vomitoria Linnaeus (Diptera: Calliphoridae) apresentaram uma maior quantidade de machos. Os resultados obtidos para os pupários grandes foram o inverso dos resultados obtidos para os pequenos, sugerindo assim, que além do tamanho há uma possível influência da diferença nutricional interespecífica dos hospedeiros sobre a razão sexual dos parasitóides.

Shuker et al. (2005) testaram uma hipótese chamada Asymetrical Local mate competition, que é a extensão da teoria de Hamilton (1967), Local mate competition (LMC), que sustenta a tendência ao aumento da taxa de fêmeas na prole de espécies onde apenas as fêmeas abandonam o pupário dispersando-se. Porém, Shuker et al. (2005) tratam de casos nos quais as fêmeas depositam seus ovos em diversos lugares, em tempos distintos, gerando emergências em tempo diferentes e encontros de machos competidores de ciclos diferentes. Neste teste, verificaram que as fêmeas de $N$. vitripennis possuem uma tendência a reduzir sua taxa de fêmeas na prole caso estejam utilizando um hospedeiro já parasitado ou caso haja um outro hospedeiro parasitado no mesmo local.

Com base no presente trabalho pode-se concluir que a densidade populacional de $N$. vitripennis afetou a duração do período pósembrionário, a razão sexual da prole e a eficiência de parasitismo. Porém, não foi observada influência sobre a produtividade dentro da amplitude trabalhada.

\section{Agradecimentos}

À Msc Ana Clara Gonçalves (Museu Nacional/UFRJ) pelo auxílio nas coletas; à Dra. Maria Angélica Penteado Dias (Departamento de Ecologia e Biologia Evolutiva da Universidade de São Carlos) pela confirmação na identificação dos parasitóides e a Fundação Rio-Zoo por ter permitido a realização de coletas, em especial ao Biólogo Anderson e ao Sr Quirino.

\section{Referências Bibliográficas}

BARBOSA, L.S. 2006. Relações quantitativas e temporárias na exposição do hospedeiro Cochliomyia macellaria (Fabricius, 1775) (Diptera: Calliphoridae) ao parasitóide Nasonia vitripennis (Walker, 1836) (Hymenoptera: Pteromalidae), em laboratório. Dissertação de mestrado, Museu Nacional/UFRJ, Rio de Janeiro.

BAUMGARTNER, D.L. \& GREENBERG, B. 1985. Distribution and medical ecology of the blow flies (Diptera: Calliphoridae) of Peru. Ann. Entomol. Soc. Am, 78(5):565-587.

CARDOSO, D. \& MILWARD-DE-AZEVEDO, E.M.V. 1995. Influência da densidade de Chrysomya megacephala (Fabricius) (Diptera: Calliphoridae) sobre a capacidade reprodutiva de fêmeas nulíparas de Nasonia vitripennis (Walker) (Hymenoptera: Pteromalidae). Rev. Bras. Entomol. 39(4):779-786.

CARDOSO, D. \& MILWARD-DE-AZEVEDO, E.M.V. 1996. Aspectos da biologia de Nasonia vitripennis (Walker) (Hymenoptera: Pteromalidae) em pupas de Chrysomya megacephala (Fabricius) e Chrysomya albiceps (Wiedemann) (Diptera: Calliphoridae), sob condições de laboratório. Rev. Bras. Entomol. 40(2):143-146.

CUNHA-E-SILVA, S.L. \& MILWARD-DE-AZEVEDO, E.M.V. 1994. Estudo comparado do desenvolvimento pós-embrionário de Cochliomyia macellaria (Fabricius) (Diptera, Calliphoridae) em duas dietas à base de carne, em laboratório. Rev. Bras. Zool. 11(4):659-668.

GUIMARÃES, J.H., PAPAVERO, N. \& PRADO, A.P. 1983. As miíases na região neotropical. Rev. Bras. Zool. 1(4):239-416.

HAMILTON, W.D. 1967. Extraordinary sex ratios. Science 156:477-488.

HARVEY, J.A. \& GOLS, G.J.Z. 1998. The influence of host quality on progeny and sex allocation in the pupal ectoparasitoid, Muscidifurax raptorellus (Hymenoptera: Pteromalidae). Bull. Entomol. Res. 88:299-304.

MAGALHÃES, M.N. \& LIMA, A.C.P. 2001. Noções de Probabilidade e Estatística. IME-USP. 3 ed. São Paulo.

MARCHIORI, C.H., SILVIA, C.G., CALDAS, E.R., ALMEIDA, K.G.S. \& CARVALHO, S.A. 2001. Primeira ocorrência do parasitóide Nasonia vitripennis (Walker) (Hymenoptera: Pteromalidae) em pupas de Peckia chrysostoma (Wiedemann) (Diptera: Sarcophagidae). Arq. Inst. Biol. 68(1):107-109.

MELLO, R.P. 2003. Chave para identificação das formas adultas das espécies da família Calliphoridae (Diptera, Brachycera, Cyclorrhapha) encontradas no Brasil. Entomol. Vect. 10(2):255-268.

MOYA-BORJA, G.E. 2003. Erradicação ou manejo integrado das miíases neotropicais das Américas. Pesq. Vet. Bras. 23:131-138.

PAES, M.J. \& MILWARD-DE-AZEVEDO E.M.V. 1998. Desenvolvimento pós-embrionário de Cochliomyia macellaria (Fabricius) (Diptera: Calliphoridae) criada em dietas naturais processadas em condições controladas. Parasitol. al Dia 22:90-96.

RUEDA, L.M. \& AXTELL, R.C. 1985. Guide to common species of pupal parasites (Hymenoptera: Pteromalidae) of the house fly and other muscoid flies associated with poultry and livestock manure. N. C. Agric. Res. Serv. Tech. Bull. 278p.

SAUNDERS, D.S., SUTTON, D. \& JARVIS, R.A. 1970. The effect of host species on diapause induction in Nasonia vitripennis. J. Insect. Physiol. 16:405-416.

SHUKER, D.M., PEN, I., DUNCAN, A.B., REECE, S.E. \& WEST, A.S. 2005 Sex Ratios under Asymetrical Local Mate Competition: Theory and a Test with Parasitoid Wasps. Amer. Naturalist. 166(3):301-316. 
Barbosa, LS. et al.

SLANSKY, J.R.F. \& SCRIBER, M. 1985. Food consumption and utilization. In. Compreensive Inss Physiol, Biochem Pharm. Oxford, Pergamon, $162 \mathrm{p}$.

WALKER, I. \& PIMENTEL, D. 1966. Correlation between maternal longevity and incidence of diapause in Nasonia vitripennis Walker (Hymenoptera: Pteromalidae). Gerontol. 12:89-98.

WHITING, A.R. 1967. The biology of the parasitic wasp Mormoniella vitripennis (= Nasonia vitripennis) (Walker). Quart. Rev. Biol. 42:333-406.
WYLIE, H.G., 1963. Some effects of host age on parasitism by Nasonia vitripennis (Walk.) (Hymenoptera: Pteromalidae). Canad. Entomol. 95:881-886

WYLIE, H.G. 1966. Survival and reproduction of Nasonia vitripennis (Walker) on different host population densities. Canad. Entomol. 98: 273-286.

Recebido em 13/09/07 Versão Reformulada recebida em 30/11/07 Publicado em 01/01/08 